\title{
Association between Sleeping Hours and Siesta and the Risk of Obesity: The SUN Mediterranean Cohort
}

\author{
Carmen Sayón-Orea ${ }^{a} \quad$ Maira Bes-Rastrollo ${ }^{a}$ Silvia Carlos ${ }^{a} \quad J u a n ~ J o s e ~ B e u n z a{ }^{a}$ \\ Francisco J. Basterra-Gortaria, b Miguel A. Martínez-González ${ }^{a}$ \\ a Department of Preventive Medicine and Public Health, University of Navarra, Pamplona, \\ ${ }^{b}$ Department of Internal Medicine (Endocrinology), Hospital Reina Sofia, Tudela, Spain
}

Key Words
Sleeping hours $\cdot$ Siesta $\cdot$ Obesity

\begin{abstract}
Objectives: Our aim was to investigate the association between sleeping hours at night and during the siesta and the incidence of obesity in a Mediterranean cohort. Methods: After a median of 6.5 years of follow-up, we included 10,532 or 9,470 participants without chronic disease or obesity at baseline for analyzing the association between the incidence of obesity and nocturnal sleep duration or having siesta. Sleeping hours and siesta were assessed at baseline. Weight was recorded at baseline and every 2 years during the follow-up. The outcome was the incidence of obesity during follow-up among participants with initial BMI $<30$ $\mathrm{kg} / \mathrm{m}^{2}$. Results: During follow-up we observed 446 new cases of obesity in the analysis of nocturnal sleep duration. Sleeping less than $5 \mathrm{~h}$ at night was associated with a higher risk of becoming obese compared to sleeping between 7 and $<8$ h (HR 1.94; 95\% CI 1.19-3.18; p for quadratic trend $=0.06$ ) after adjusting for potential confounders. During follow-up, we observed 396 incident cases of obesity in the analysis of siesta. Those who took a siesta for 30 $\mathrm{min} /$ day had a $33 \%$ lower risk of becoming obese (HR 0.67; 95\% CI 0.46-0.96; $\mathrm{p}$ for quadratic trend $=0.13$ ) compared to those who did not take siesta. Conclusion: Our results suggest that short nocturnal sleep duration could be a modifiable risk factor for obesity. It is possible that this association may be stronger among men and subjects who experienced previous weight gain. Additionally, siesta might be a novel and independent protective factor for obesity; however, confirmatory studies are needed.


Sayón-Orea et al.: Association between Sleeping Hours and Siesta and the Risk of Obesity: The SUN Mediterranean Cohort

\section{Introduction}

Obesity has become a worldwide epidemic; it is a health concern in both developed and developing countries. According to the World Health Organization, obesity has more than doubled since 1980 and it is the fifth leading risk for global deaths [1]. The fundamental cause of obesity is an energy imbalance between calories consumed and calories expended. Although diet and physical activity play an important role in the risk of obesity, an additional and novel factor may be insufficient sleep [2]. It has been demonstrated that reduction in sleep duration or poor sleep quality can alter appetite, satiety, and energy balance $[3,4]$. Consequently, it is thought that alterations in sleep pattern could have an important role in the obesity epidemic.

In the last years, results of epidemiological (cross-sectional) studies among adults about short sleep duration and obesity are not consistent as they reported either no association [5], a U-shaped association [6], or a positive association [7-12]. However, the causal direction has been difficult to establish in these cross-sectional studies due to their design. Prospective studies evaluating this hypothesis found an inverse association between short sleep duration and future weight gain or the risk of obesity [7, 13-20]. Nevertheless, inconsistencies are reported in two studies $[16,17]$, and the association between short sleep duration and future weight gain appeared to decrease with increasing age in one prospective study [20] and one cross-sectional study [5]. In addition, one study showed that long sleep duration was also associated with obesity [18].

Afternoon sleep, midday nap, or siesta is a common practice, particularly in many Mediterranean countries including Spain. Null epidemiological evidence exists about the relationship between siesta and the risk of obesity or weight gain in adults. As there is no previous cross-sectional or longitudinal assessment about siesta and the incidence of obesity among adults, here we present the first prospective study that evaluates this association.

Therefore, the aim of our study was to investigate the association between nocturnal sleep duration and siesta with the incidence of obesity in a large Mediterranean cohort.

\section{Subjects and Methods}

\section{Study Population}

The SUN study is a prospective, multipurpose and dynamic Spanish cohort, designed to establish associations between diet and the occurrence of several diseases including obesity [21]. The participants were followed up every 2 years using mailed questionnaires. A description of the study methods was published elsewhere [21]. Briefly, participants' recruitment started in December 1999, and it is permanently open. All participants are university graduates, where the age ranged from 20 to 90 years. Up to March 2009 the data set of the SUN project included 20,066 participants. For the present analyses, we excluded those participants with prevalent obesity at baseline $(\mathrm{n}=968)$, those who reported excessively low or high values for total energy intake (less than $800 \mathrm{kcal} /$ day in men and $500 \mathrm{kcal} /$ day in women or more than 4,000 kcal/day in men and 3,500 kcal/day in women; $n=1,831)$, women who were pregnant $(n=2,267)$, and those who reported chronic diseases at baseline (diabetes, cancer and cardiovascular disease (CVD); $n=1,521$ ), leaving a total of 13,479 participants. The rationale for these exclusions was to avoid or reduce information bias as it has been recommended in nutritional epidemiology [22]. Additionally we had 1,434 participants who were lost to follow-up leaving a total of 12,045 (retention rate 89\%). From this group, we excluded another 1,513 participants who did not answer the question of how many hours they sleep at night, leaving a total of 10,532 participants available for the analysis of the relationship between obesity and nocturnal sleep duration (fig. 1), or another 2,575 participants who did not answer the question of siesta leaving a total of 9,470 participants available for the analysis of the association between obesity and siesta.

The Institutional Review Board of the University of Navarra approved the study protocol. Voluntary completion of the first self-administrated questionnaire was considered to imply informed consent. 
Sayón-Orea et al:: Association between Sleeping Hours and Siesta and the Risk of Obesity: The SUN Mediterranean Cohort

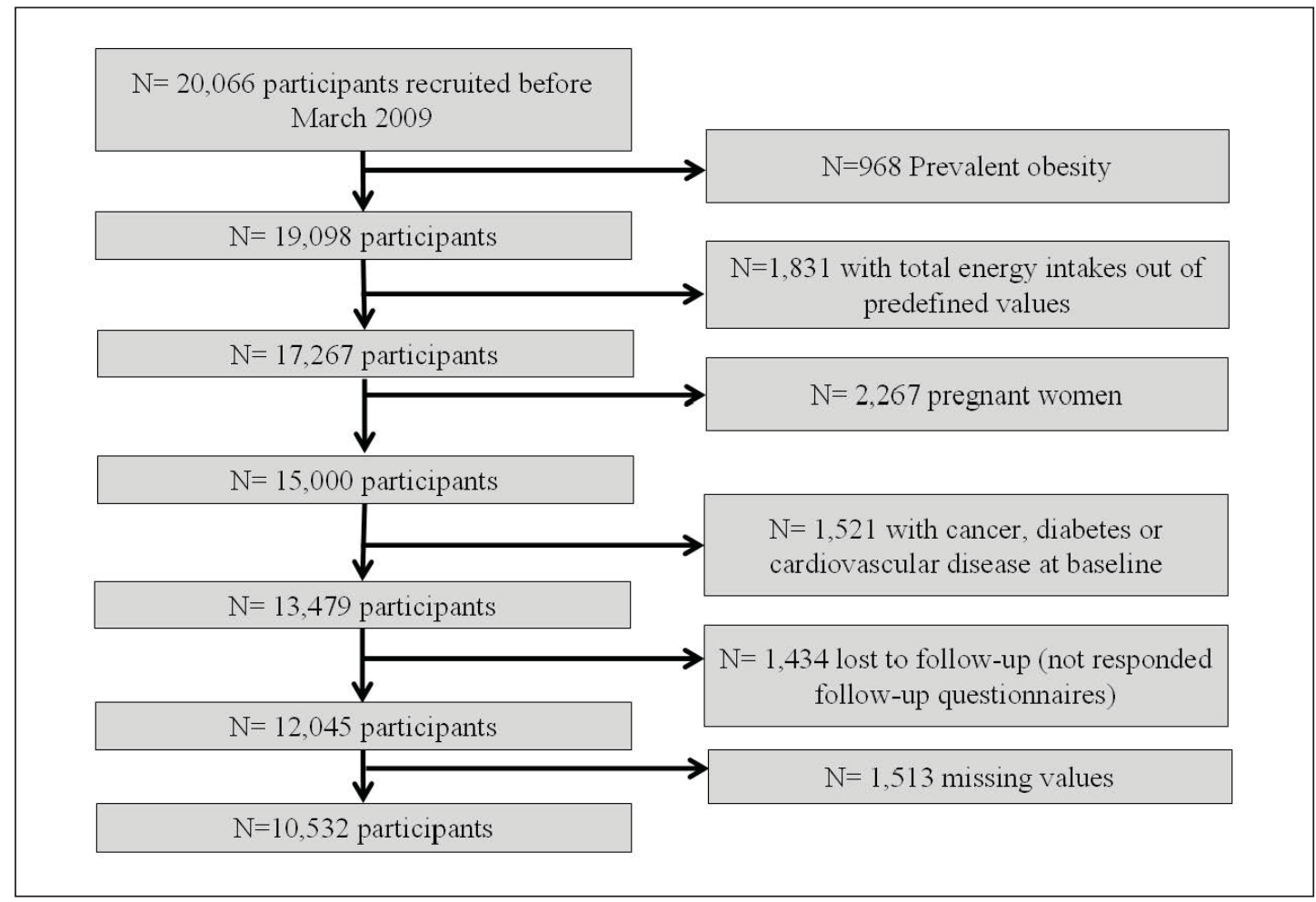

Fig. 1. Flow chart of participants.

\section{Sleep and Siesta Duration}

Average nocturnal sleep duration was obtained by adding the information from the following two questions included in the baseline questionnaire: 'How many hours per day do you sleep at night during the weekdays?' 'And during the weekends?' We multiplied the first number of hours by 5 and the second by 2 and then divided the total by 7 . Average siesta duration was similarly calculated using these two questions: 'How many hours per day do you take a siesta during the weekdays?' 'And during the weekends?' The information about sleeping hours and siesta was validated in a comparison versus prospective records in a subsample of participants (intraclass correlation coefficients 0.65 and 0.68 for sleeping hours and siesta, respectively).

\section{Dietary Assessment}

At baseline, food intake was assessed by a semiquantitative food frequency questionnaire (FFQ) previously validated in Spain with 136 food items $[23,24]$. The questionnaire was based on standard portion sizes where frequencies were measured in nine categories (ranging from never/almost never to at least 6 times/ day) for each food item.

A trained dietitian updated the nutrient databank using available information on food composition tables for Spain $[25,26]$.

\section{Assessment of Confounders}

The baseline questionnaire also included other questions (46 items for men and 54 for women) that assessed the participants' medical history (prevalence of chronic diseases such as cancer, diabetes, and CVD), health-related habits (smoking status, physical activity during leisure time), lifestyle, and sociodemographic variables (sex, age, marital status, and employment) as well as anthropometric data (weight and height).

Outcome Assessment

Participants' weight was recorded at baseline and during the follow-up questionnaires. The median follow-up time for these analyses was 6.5 years. The outcome in the current analysis was the incidence of 
Sayón-Orea et al:: Association between Sleeping Hours and Siesta and the Risk of Obesity: The SUN Mediterranean Cohort

obesity in the cohort (participants with a baseline BMI value lower than $30 \mathrm{~kg} / \mathrm{m}^{2}$ and with a BMI $\geq 30 \mathrm{~kg} /$ $\mathrm{m}^{2}$ after follow-up). BMI was calculated as the self-reported weight in kilograms divided by the square of height in meters. The validity and reproducibility of self-reported weights were assessed in a subsample of the cohort. The mean relative error in self-reported weight was $1.45 \%$, and the correlation coefficient between measured and self-reported weight was 0.99 (95\% confidence interval (CI) 0.98-0.99) [27]. To quantify the amount of physical activity, we inquired about 17 activities, and a metabolic equivalent index (MET-h/week) was computed. The MET-h/week, showed to adequately correlate with the objectively measured energy expenditure in a previous validation in a subsample of the cohort (Spearman's $\rho=0.51$; 95\% CI 0.232-0.707) [28]. The validation studies were performed on the baseline information.

\section{Statistical Analyses}

Participants were classified into four categories according to the average of hours they slept at night: $<5 \mathrm{~h}, 5$ to $<7 \mathrm{~h}, 7$ to $<8 \mathrm{~h}$, and $\geq 8 \mathrm{~h}$; or according to the time they took a siesta: 'never or almost never', those who took a typical siesta of $30 \mathrm{~min}$, those who took $>30 \mathrm{~min}$ to $<1 \mathrm{~h}$, and those who took $\geq 1 \mathrm{~h}$. Additionally, we calculated the total daily sleep duration by summing up the hours participants reported to sleep at night and the time they took a siesta, and we classified them into the same four categories. We considered for all analyses sleeping between 7 and $<8 \mathrm{~h}$ as the reference category in the nocturnal sleep duration analysis, and those who 'never or almost never' took a siesta as the reference category in the siesta analysis.

In addition, to assess the joined effect of nocturnal sleep duration and siesta we have cross-classified both exposures, taking as the reference category sleeping from 7 to $<8 \mathrm{~h}$ at night and taking a 30 -min siesta.

Cox regression modeling was used to estimate the hazard ratio (HR) for the development of obesity during the follow-up. HR and the 95\% CI were calculated. Some previous cross-sectional and prospective studies found that the association between sleeping hours and the risk of incident obesity did not follow a linear trend; instead a U-shaped association was found. Therefore, we used the quadratic term to better account for this departure from linearity.

We fitted a crude univariable model, an age- and sex-adjusted model, and two multivariable models. The first model was adjusted for age, sex, physical activity (MET-h/week), smoking status (non-smoker, smoker, former smoker), sitting (h/day), total energy intake (kcal/day), consumption of sugared-soft drinks (ml/ day), fast food consumption (g/day), snacking between meals (yes/no), caffeine intake (mg/day), alcohol intake (ml/day), regular snorer (yes/no), insomnia (yes/no), and siesta (yes/no) or categories of sleeping hours ( $<5 \mathrm{~h}, 5$ to $<7 \mathrm{~h}, 7$ to $<8 \mathrm{~h}$, and $\geq 8 \mathrm{~h}$ ). The second multivariable model was additionally adjusted for baseline BMI $\left(\mathrm{kg} / \mathrm{m}^{2}\right)$. According to the current recommendations [29] to select potential confounding factors, we took into account previously published scientific literature on causal risk factors for weight gain [30]. In addition we adjusted for some variables strongly associated with lifestyles such as smoking.

We evaluated the interaction of sex and previous weight gain $(<3 \mathrm{~kg}$ and $\geq 3 \mathrm{~kg}$ in the 5 years previous to baseline assessment) with the categories of nocturnal sleep duration through likelihood ratio tests for the product term introduced in fully adjusted models, and we conducted stratified analyses in both cases.

All $p$ values presented are two-tailed; $p<0.05$ was considered statistically significant. Analyses were performed using SPSS version 15.0 (SPSS Inc. Chicago, IL, USA).

\section{Results}

\section{Sleeping Hours}

The main characteristics of participants according to nocturnal sleep duration are presented in table 1 . The mean age of the participants was $39 \pm 12$ years, and the mean BMI was $23.4 \pm 2.9 \mathrm{~kg} / \mathrm{m}^{2}$. Comparing participants in the category of sleeping $<5 \mathrm{~h}$ and the reference category ( 7 to $<8 \mathrm{~h}$ ), participants belonging to the reference category were younger, had a lower caffeine intake, showed lower proportions of insomnia and regular snoring, and were found of having siesta more frequently. Other baseline characteristics were very similar in all categories.

During the follow-up period (median of 6.5 years), we observed 446 new (incident) cases of obesity. Sleeping $<5 \mathrm{~h}$ at night was associated with a significantly greater risk of becoming obese compared with sleeping between 7 and $<8 \mathrm{~h}$ at night (HR 1.94 (1.19-3.18)), and the HR 
Sayón-Orea et al.: Association between Sleeping Hours and Siesta and the Risk of Obesity: The SUN Mediterranean Cohort

Table 1. Baseline characteristics of participants according to sleeping hours/day: the SUN cohort 1999$2011^{\mathrm{a}}$

\begin{tabular}{|c|c|c|c|c|}
\hline & \multicolumn{4}{|c|}{ Sleeping hours/day } \\
\hline & $<5$ & 5 to $<7$ & 7 to $<8$ & $\geq 8$ \\
\hline $\mathrm{N}$ & 304 & 2,391 & 4,770 & 3,067 \\
\hline Age, years & $42.8(13.7)$ & $40.9(12.5)$ & $39.4(10.8)$ & $37.0(11.2)$ \\
\hline Men, $\%$ & 46.7 & 48.6 & 47.1 & 37.1 \\
\hline $\mathrm{BMI}, \mathrm{kg} / \mathrm{m}^{2}$ & $23.7(2.9)$ & $23.8(2.9)$ & $23.4(2.8)$ & $23.0(2.9)$ \\
\hline Regular snorer, \% & 20.7 & 21.6 & 17.9 & 15.2 \\
\hline Insomnia, \% & $5.8(2.0)$ & $5.6(2.1)$ & $5.7(2.1)$ & $5.7(2.1)$ \\
\hline Siesta, \% & 57.2 & 59.1 & 60.6 & 59.8 \\
\hline Previous weight gain, $\%{ }^{\mathrm{b}}$ & 42.1 & 33.9 & 34.1 & 34.0 \\
\hline Physical activity, MET-s/week & $20.6(24.2)$ & $21.7(24.3)$ & $21.3(21.3)$ & $20.9(22.7)$ \\
\hline Sitting, h/day & $5.8(2.0)$ & $5.6(2.1)$ & $5.7(2.1)$ & $5.7(2.1)$ \\
\hline Current smokers, \% & 24.0 & 23.7 & 21.7 & 21.6 \\
\hline Former smokers, $\%$ & 28.6 & 30.6 & 30.3 & 29.0 \\
\hline Total energy intake, kcal/day & $2,319(687)$ & $2,345(650)$ & $2,384(611)$ & 2,365 (599) \\
\hline Carbohydrate intake, $\%$ of energy & $44.1(8.1)$ & $43.6(7.5)$ & $43.5(7.1)$ & $43.6(7.3)$ \\
\hline Protein intake, $\%$ of energy & $17.8(4.0)$ & $18.2(3.3)$ & $17.9(3.1)$ & $17.9(3.2)$ \\
\hline Total fat intake, \% of energy & $35.7(7.8)$ & $35.8(6.7)$ & $36.4(6.2)$ & $36.7(6.6)$ \\
\hline Alcohol intake, \% of energy & $2.4(3.7)$ & $2.3(3.6)$ & $2.2(2.9)$ & $1.8(2.6)$ \\
\hline Fast food, g/day & $18.3(20.9)$ & $20.1(22.8)$ & $21.3(19.4)$ & $21.3(19.6)$ \\
\hline Sugared-soft drinks, ml/day & $61.8(146.6)$ & $61.5(132.9)$ & $61.0(114.0)$ & $62.2(132.3)$ \\
\hline Snacking between meals, \% & 33.2 & 32.5 & 30.0 & 32.2 \\
\hline Caffeine, mg/day & $43.5(44.6)$ & $46.0(44.5)$ & $44.7(38.5)$ & $39.7(37.7)$ \\
\hline
\end{tabular}

${ }^{a}$ Values are expressed as mean (SD), unless otherwise stated.

$\mathrm{b} \geq 3 \mathrm{~kg}$ in the 5 years before the baseline assessment.

for sleeping $\geq 8 \mathrm{~h}$ was $1.13(0.89-1.43)$ ( $\mathrm{p}$ for quadratic trend $=0.06$ ), after adjusting for potential confounders (table 2). However, when nocturnal sleep duration was entered in the model as a continuous variable, the quadratic trend was not significant in the multivariableadjusted model (results not shown in the tables).

We split the database according to weight gain (no weight gain or $<3 \mathrm{~kg}$ and $\geq 3 \mathrm{~kg}$ ) before enrollment ( 5 years previous to the baseline questionnaire) and analyzed the relationship between nocturnal sleep duration and subsequent risk of becoming obese within these two categories. The HRs for incident obesity in all categories of nocturnal sleep duration were significantly higher in the group of participants who were gaining weight before the baseline questionnaire ( $<5$ h: HR 3.36 (1.82-6.21); 5 to $<7$ h: HR 1.43 (1.03-1.98), 7 to <8 h: HR 1.00 (ref.), and $\geq 8$ h: HR 1.39 (1.00-1.93); p for quadratic trend 0.001) than in the group who did not gain weight or gained less than $3 \mathrm{~kg}(<5 \mathrm{~h}$ : HR 1.03 (0.40-2.61); 5 to $<7 \mathrm{~h}$ : HR $0.99(0.70-$ 1.39), 7 to $<8$ h: HR 1.00 (ref.) and $\geq 8$ h: HR 0.95 (0.67-1.38); p for quadratic trend 0.99 ), finding a statistically significant interaction $p=0.03$ (table 3 ). We also evaluated other possible interactions to determine whether the association between sleeping hours and the risk of obesity depended on sex. The risk of becoming obese associated with very low sleeping hours at night was higher among men than among women only in the category of sleeping $<5$ $\mathrm{h}$ at night. The estimates for sleeping $<5 \mathrm{~h}$ were HR 2.09 (1.18-3.69) $\mathrm{p}$ for quadratic trend $=$ 0.05 for men and HR $1.26(0.44-3.57) \mathrm{p}$ for quadratic trend $=0.30$ for women, resulting in a $\mathrm{p}$ for interaction $=0.056($ table 4$)$. 
Sayón-Orea et al.: Association between Sleeping Hours and Siesta and the Risk of Obesity: The SUN Mediterranean Cohort

Table 2. HR and 95\% CI for incident obesity during the follow-up in 10,532 participants according to sleeping hours/day: the SUN cohort 1999-2011

\begin{tabular}{|c|c|c|c|c|c|}
\hline & \multicolumn{5}{|c|}{ Sleeping hours/day } \\
\hline & $<5$ & 5 to $<7$ & 7 to $<8$ & $\geq 8$ & $\begin{array}{l}\mathrm{p} \\
\text { quadratic trend }\end{array}$ \\
\hline Person years & 2,035 & 15,546 & 31,363 & 20,468 & \\
\hline Incident cases & 19 & 123 & 187 & 117 & \\
\hline Crude HR (95\% CI) & $1.58(0.99-2.54)$ & $1.34(1.07-1.68)$ & 1.00 Ref. & $0.96(0.76-1.21)$ & 0.01 \\
\hline Age-sex-adjusted HR (95\% CI) & $1.60(1.00-2.57)$ & $1.33(1.06-1.67)$ & 1.00 Ref. & $1.04(0.82-1.31)$ & 0.03 \\
\hline Multivariable $\mathrm{HR}^{\mathrm{a}}(95 \% \mathrm{CI})$ & $1.54(0.95-2.49)$ & $1.25(0.99-1.58)$ & 1.00 Ref. & $1.06(0.84-1.33)$ & 0.13 \\
\hline Multivariable $\mathrm{HR}^{\mathrm{b}}$ (95\% CI) & $1.94(1.19-3.18)$ & $1.15(0.91-1.44)$ & 1.00 Ref. & $1.13(0.89-1.43)$ & 0.06 \\
\hline
\end{tabular}

${ }^{a}$ Adjusted for age, sex, smoking status, physical activity, time spent sitting down, fast food, sugared soft drinks, snacking between meals, total energy intake, caffeine, alcohol, snoring, insomnia and siesta hours.

${ }^{\mathrm{b}}$ Additionally adjusted for baseline BMI.

Table 3. HR and 95\% CI for incident obesity during the follow-up in 10,532 participants according to sleeping hours/day, stratified by previous 5-year weight gain: the SUN cohort 1999-2011

\begin{tabular}{llll} 
Sleeping hours/day & & & \\
\hline$<5$ & 5 to $<7$ & 7 to $<8 \quad \geq 8$ & $\mathrm{p}$ \\
& & & quadratic trend
\end{tabular}

Weight gain $<3 k g$

Person years

Incident cases

Crude HR $(95 \% \mathrm{CI})$

Age-sex-adjusted HR (95\% CI)

1,222

5

Multivariable $\mathrm{HR}^{\mathrm{a}}(95 \% \mathrm{CI})$

Multivariable $\mathrm{HR}^{\mathrm{b}}(95 \% \mathrm{CI})$
$0.89(0.36-2.20)$

$0.89(0.36-2.19)$

$0.83(0.34-2.07)$

$1.03(0.40-2.61)$
10,328

56

$1.18(0.50-1.65)$

$1.15(0.83-1.61)$

$1.06(0.76-1.48)$

$0.99(0.70-1.39)$

\section{$20,623 \quad 13,477$}

$95 \quad 52$

1.00 Ref. $\quad 0.84(0.60-1.18) \quad 0.37$

1.00 Ref. $\quad 0.93(0.66-1.30) \quad 0.70$

1.00 Ref. $\quad 0.91(0.65-1.28) \quad 0.85$

1.00 Ref. $0.95(0.67-1.38) \quad 0.99$

\section{Weight gain $\geq 3 \mathrm{~kg}$}

Person years

Incident cases

Crude HR $(95 \% \mathrm{CI})$

Age-sex-adjusted HR (95\% CI)

Multivariable $\mathrm{HR}^{\mathrm{a}}(95 \% \mathrm{CI})$

$\begin{array}{ll}813 & 5,218 \\ 14 & 67 \\ 2.06(1.17-3.61) & 1.53(1.14-2.09) \\ 2.09(1.19-3.67) & 1.53(1.12-2.10) \\ 2.05(1.14-3.68) & 1.48(1.08-2.04) \\ 3.36(1.82-6.21) & 1.43(1.03-1.98)\end{array}$

$\begin{array}{lll}10,741 & 6,991 & \\ 92 & 65 & \\ 1.00 \text { Ref. } & 1.08(0.79-1.49) & 0.009 \\ 1.00 \text { Ref. } & 1.15(0.84-1.59) & 0.01 \\ \text { 1.00 Ref. } & 1.22(0.89-1.68) & 0.03 \\ 1.00 \text { Ref. } & 1.39(1.00-1.93) & 0.001\end{array}$

Multivariable $\mathrm{HR}^{\mathrm{b}}(95 \% \mathrm{CI})$

${ }^{a}$ Adjusted for age, sex, smoking status, physical activity, time spent sitting down, fast food, sugared soft drinks, snacking between meals, total energy intake, caffeine, alcohol, snoring, insomnia and siesta hours.

${ }^{\mathrm{b}}$ Additionally adjusted for baseline BMI.

We conducted a secondary analysis including those participants who initially were excluded because they had low or high energy reports according to published recommendations in nutritional epidemiology [22], and the HRs were: 1.58 (0.97-2.57), 1.15 (0.92-1.44), 1.00 (ref.) and $1.10(0.88-1.39)$ for $<5 \mathrm{~h}, 5$ to $<7 \mathrm{~h}, 7$ to $<8 \mathrm{~h}$ and $\geq 8 \mathrm{~h}$, respectively (results not shown in the tables). 
Sayón-Orea et al.: Association between Sleeping Hours and Siesta and the Risk of Obesity: The SUN Mediterranean Cohort

Table 4. HR and 95\% CI for incident obesity during the follow-up in 10,532 participants according to sleeping hours/day, stratified by sex: the SUN cohort 1999-2011

$\begin{array}{llll}\text { Sleeping hours/day } & & \\ <5 & 5 \text { to }<7 & 7 \text { to }<8 \quad \geq 8 & \text { p quadratic trend }\end{array}$

\section{Men}

Person years

Incident cases

Crude HR (95\% CI)

Age-sex-adjusted HR (95\% CI)

Multivariable $\mathrm{HR}^{\mathrm{a}}(95 \% \mathrm{CI})$

Multivariable $\mathrm{HR}^{\mathrm{b}}(95 \% \mathrm{CI})$
912

15

$2.04(1.20-3.49)$

$1.98(1.16-3.39)$

$1.91(1.10-3.32)$

$2.09(1.18-3.69)$
7,648

77

$1.24(0.93-1.64)$

$1.22(0.91-1.62)$

$1.18(0.88-1.57)$

$1.03(0.91-1.45)$
15,481

127

1.00 Ref. $\quad 0.88(0.65-1.20) \quad 0.01$

1.00 Ref. $\quad 0.90(0.66-1.22) \quad 0.03$

1.00 Ref. $\quad 0.90(0.66-1.23) \quad 0.06$

1.00 Ref. $\quad 0.88(0.64-1.21) \quad 0.05$

\section{Women}

Person years

Incident cases

Crude HR (95\% CI)

Age-sex-adjusted HR (95\% CI)

Multivariable $\mathrm{HR}^{\mathrm{a}}(95 \% \mathrm{CI})$

Multivariable $\mathrm{HR}^{\mathrm{b}}(95 \% \mathrm{CI})$

7,898
46
$1.58(1.07-2.32)$
$1.58(1.07-2.32)$
$1.43(0.97-2.12)$
$1.34(0.90-2.00)$

15,881

$15,881-12,461$

$60 \quad 59$

1.00 Ref. $1.27(0.89-1.82) \quad 0.13$

1.00 Ref. $1.28(0.89-1.84) \quad 0.13$

1.00 Ref. $1.32(0.92-1.90) \quad 0.25$

1.00 Ref. $1.43(0.97-2.10) \quad 0.30$

${ }^{a}$ Adjusted for age, smoking status, physical activity, time spent sitting down, fast food, sugared soft drinks, snacking between meals, total energy intake, caffeine, alcohol, snoring, insomnia and siesta hours.

${ }^{\mathrm{b}}$ Additionally adjusted for baseline BMI.

When we analyzed the total daily sleep duration and the risk of developing obesity, we found that the association decreased but remained statistically significant for sleeping $<5$ h/day ( $<5$ h: HR 1.70 (1.00-3.09); 5 to <7 h: HR 1.17 (0.91-1.50), 7 to <8 h: HR 1.00 (ref.) and $\geq 8$ h: HR $1.08(0.86-1.35)$; p for quadratic trend $=0.21$ ) (results not shown in the tables).

\section{Siesta}

During the follow-up period, we observed 396 new (incident) cases of obesity.

Those who took a siesta for $30 \mathrm{~min} /$ day had a lower risk of becoming obese (HR 0.67 $(0.46-0.96)$; $p$ for quadratic trend $=0.13$ ) compared to those who never or almost never took a siesta. A siesta of more than 30 min did not show this protective effect: $>30$ min to $<1 \mathrm{~h}$ : HR $0.92(0.72-1.18)$ and $\geq 1$ h: HR $0.78(0.51-1.19)$ (table 5).

We repeated the analysis without any exclusion because of energy intake. The HRs were 0.68 (0.48-0.95), $0.93(0.74-1.18)$ and $0.75(0.49-1.13)$ for $30 \mathrm{~min},>30 \mathrm{~min}$ to $<1 \mathrm{~h}$ and $\geq 1$ h duration of siesta, respectively (reference: never or almost never having siesta) that included all subjects irrespective of their energy intake (results not shown in the tables).

\section{Nocturnal Sleep Duration and Siesta}

When we analyzed the cross-stratified associations of nocturnal sleep duration and siesta, sleeping $<7 \mathrm{~h}$ at night and taking a siesta of more than 30 min was associated with a higher risk of becoming obese during the follow-up in comparison with sleeping 7 to $<8 \mathrm{~h}$ and taking a siesta of $30 \mathrm{~min}$ (HR 2.02 (1.12-3.67)) (table 6). 
Sayón-Orea et al:: Association between Sleeping Hours and Siesta and the Risk of Obesity: The SUN Mediterranean Cohort

Table 5. HR and $95 \%$ CI for incident obesity during the follow-up in 9,470 participants according to siesta minutes/day: the SUN cohort 1999-2011

\begin{tabular}{|c|c|c|c|c|c|}
\hline & \multicolumn{5}{|c|}{ Siesta minutes/day } \\
\hline & $\begin{array}{l}\text { never / } \\
\text { almost never }\end{array}$ & $30 \mathrm{~min}$ & $>30 \mathrm{~min}$ to $<1 \mathrm{~h}$ & $\geq 1 \mathrm{~h}$ & $\begin{array}{l}\mathrm{p} \\
\text { quadratic trend }\end{array}$ \\
\hline Person years & 37,005 & 7,745 & 14,432 & 3,140 & \\
\hline Incident cases & 245 & 35 & 92 & 24 & \\
\hline Crude HR (95\% CI) & 1.00 Ref. & $0.69(0.48-0.98)$ & $0.98(0.77-1.24)$ & $1.16(0.76-1.76)$ & 0.17 \\
\hline Age-sex-adjusted HR (95\% CI) & 1.00 Ref. & $0.64(0.45-0.92)$ & $0.92(0.72-1.16)$ & $1.19(0.78-1.81)$ & 0.06 \\
\hline Multivariable $\mathrm{HR}^{\mathrm{a}}(95 \% \mathrm{CI})$ & 1.00 Ref. & $0.67(0.46-0.96)$ & $0.92(0.72-1.18)$ & $0.78(0.51-1.19)$ & 0.13 \\
\hline
\end{tabular}

${ }^{a}$ Adjusted for age, sex, baseline BMI, smoking status, physical activity, time spent sitting down, fast food, sugared soft drinks, snacking between meals, total energy intake, caffeine, alcohol, snoring, insomnia and sleeping categories.

Table 6. Multivariable HR and 95\% CI for incident obesity during follow-up in 9,470 participants according to their cross-stratified classification by nocturnal sleep duration and siesta: the SUN cohort 1999-2011 a

\begin{tabular}{llll}
\hline Siesta duration & \multicolumn{2}{l}{ Nocturnal sleep duration, $h$} & $\geq 8$ \\
\cline { 2 - 4 } & $<7$ & 7 to $<8$ & $0.98(0.44-2.18)$ \\
\hline $30 \mathrm{~min}$ & $1.28(0.52-3.15)$ & 1.00 Ref. & $1.68(0.96-2.94)$ \\
never or almost never & $1.58(0.90-2.78)$ & $1.61(0.95-2.72)$ & $1.57(0.89-2.75)$ \\
$>30$ min & $2.02(1.12-3.67)$ & $1.19(0.66-2.16)$ & \\
\hline
\end{tabular}

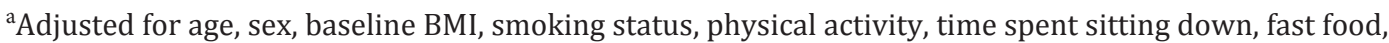
sugared soft drinks, snacking between meals, total energy intake, caffeine, alcohol, snoring, and insomnia.

\section{Discussion}

The results of this prospective study showed that short sleep duration at night $(<5 \mathrm{~h})$ was associated with a higher risk of obesity; additionally, a siesta of 30 min was inversely associated with the risk of obesity.

Obesity and short sleep duration might be linked [31]. Two mechanisms could account for potential causal pathways between reduced sleep hours and obesity: i) sleep loss has an impact on the hormones involved in appetite regulation; lower levels of leptine (appetiteinhibiting hormone) and higher levels of ghrelin (appetite-stimulating hormone) are associated with less sleeping hours [3, 4]; ii) less time sleeping means more opportunities to eat. Therefore, reduced sleep could lead to increased food intake and increased appetite that could lead to obesity. Moreover, sleepiness and fatigue may result in reduced energy expenditure through decreased physical activity [32], even though in this cohort study all participants had almost the same physical activity (MET-h/week).

Recent cross-sectional and prospective epidemiological studies found that reduced sleep duration is associated with a major risk of developing obesity in children [12-16] and in adults $[8,10,11,13-15]$. Our study is consistent with these previous studies.

We split our database according to the weight gained in the previous 5 years of the baseline questionnaire in two groups: those who gained 3 or more $\mathrm{kg}$, and those who did not 
Sayón-Orea et al.: Association between Sleeping Hours and Siesta and the Risk of Obesity: The SUN Mediterranean Cohort

gain weight or gained less than $3 \mathrm{~kg}$. We found that those who were gaining weight before enrollment were those with the higher subsequent risk of obesity. We observed the association between sleeping hours and the risk of obesity in all categories; however, the strongest association was found among those who were gaining weight before enrollment, especially when comparing sleeping $<5 \mathrm{~h}$ with sleeping between 7 and $<8 \mathrm{~h}$ at night. In addition, the $\mathrm{p}$ value for interaction was significant. Therefore, we think that our study does not support a causal association between nocturnal sleeping duration and obesity among participants with stable weight. This differential effect in this group of participants could be attributed to other lifestyle or genetic factors that render them susceptible to subsequent weight gain. This effect modification according to previous weight gain has been observed in our cohort in previous analyses on other risk factors for obesity [33].

We found some differences between sexes, but these might be a result of limited power due to a lower obesity incidence in women or even may also be explained by other factors such as an overall healthier lifestyle in women. Thus, our results on this particular issue should be confirmed in future studies.

Regarding siesta analysis, to our knowledge this is the first time that this finding (an association between siesta and a reduced risk of obesity in adults) is reported in a large prospective study. The proportion of participants taking siesta among those who slept less than $5 \mathrm{~h}$ was lower than among those who slept 7 to $<8 \mathrm{~h}$ at night. We found a protective effect associated with taking a 30-min siesta during the day compared to not taking siesta during the day. In contrast, one study in children has found that day time sleep had a little effect on subsequent obesity [34]. Some studies in adults have assessed other different associations for siesta, for example inverse associations between siesta and CVD have been reported [35]. Also, a positive association between siesta and overall mortality has been reported in elderly people [36]. However, a 33\% reduction of the risk of developing obesity in our study deserves further research to clarify the possible mechanisms by which siesta might protect against the development of obesity.

When we analyzed the combined effect of nocturnal sleep duration and having a siesta, we found that sleeping less than $7 \mathrm{~h}$ and taking a siesta of more than 30 min were associated with a higher risk of incident obesity in comparison with sleeping 7 to $>8 \mathrm{~h}$ and taking a 30-min siesta. With this result in mind, it could be thought that having a siesta did not avoid the higher risk of obesity observed among participants sleeping $<7 \mathrm{~h}$; however those participants who took a siesta of $30 \mathrm{~min}$ had the lowest risk among those who slept $<7 \mathrm{~h}$. Previous studies have observed that a short siesta (less than $30 \mathrm{~min}$ ) is associated with several benefits (promotes wakefulness and enhances performance and learning abilities), but in contrast, longer naps are associated with adverse long-term health effects (higher morbidity and mortality) [37].

Our study has many strengths. First, there is the prospective design which eludes the possibility of reverse causation bias present in cross-sectional studies. Other strengths are the use of a wide range of scoring for each food portion ( 9 categories) and the previous validation of the methods used to assess the main variables such as weight and BMI [27], physical activity [28], and the FFQ [23, 24] as well as the large number of participants included in the cohort. It might be thought that a potential limitation of our study is that it only included university graduates and therefore, is not representative of the general Spanish population. This issue may affect the generalizability of our findings; therefore, we have to be cautious in extrapolating these results to the general population. However, it could also have actually enhanced the internal validity of our study because the high level of education and the homogeneity of the cohort reduced the potential confounding related to socioeconomic status. In addition, the high educational level of our participants provides us a better quality in the information. 
A potential limitation of our study is the self-reported exposure and outcome. A tendency for participants to underestimate their weight and overestimate their height may have affected our results. However, self-reported weight and BMI was previously validated in a subsample of the cohort showing a very high reproducibility [27].

\section{Conclusion}

Our results suggest that short nocturnal sleep duration could be a modifiable risk factor for obesity. It is possible that this association may be stronger among men and subjects who experienced previous weight gain. Additionally, siesta might be a novel and independent protective factor for obesity; however, confirmatory studies are needed.

\section{Acknowledgements}

We thank participants of the SUN project for their continued cooperation and participation. We thank all the members of the SUN project for administrative, technical and material support. MAM-G helped to design the study and collect the data. MB-R and CS-O analyzed the data and drafted the manuscript. All authors edited and critically reviewed the manuscript. None of the authors had any conflicts of interest.

The SUN Study has received funding from the Spanish Government (Grants PI01/0619, PI030678, PI040233, PI042241, PI050976, PI070240, PI070312, PI081943, PI080819, PI1002658, PI1002293, RD06/0045, G03/140 and 87/2010), the Navarra Regional Government (36/2001, 43/2002, 41/2005, $36 / 2008,45 / 2011$ ) and the University of Navarra.

\section{Disclosure Statement}

The authors declare no conflict of interest.

\section{References}

1 WHO: Obesity and Overweight. Report 311. Geneva, WHO, 2011.

Knutson K: Does inadequate sleep play a role in vulnerability to obesity? Am J Hum Biol 2012;24:361-371.

-3 Spiegel K, Tasali E, Penev P, Van Cauter E: Brief communication: Sleep curtailment in healthy young men is associated with decreased leptin levels, elevated ghrelin levels, and increased hunger and appetite. Ann Intern Med 2004;141:846-850.

4 Spiegel K, Leproult R, L'hermite-Balriaux M, Copinschi G, Penev P, Van Cauter E: Leptin levels are dependent on sleep duration: relationships with sympathovagal balance, carbohydrate regulation, cortisol, and thyrotropin. J Clin Endocrinol Metab 2004;89:5762-5771.

5 Lauderdale D, Knutson K, Yan L, Rathouz P, Hulley S, Sidney S, et al: Objectively measured sleep characteristics among early-middle-aged adults: the CARDIA study. Am J Epidemiol 2006;164:5-16.

6 Taheri S, Lin L, Austin D, Young T, Mignot E: Short sleep duration is associated with reduced leptin, elevated ghrelin, and increased body mass index. PLoS Med 2004;1:e62-e62.

7 Gangwisch J, Malaspina D, Boden Albala B, Heymsfield S: Inadequate sleep as a risk factor for obesity: analyses of the NHANES I. Sleep 2005;28:1289-1296.

-8 Vioque J, Torres A, Quiles J: Time spent watching television, sleep duration and obesity in adults living in Valencia, Spain. Int J Obes 2000;24:1683-1688.

-9 Cournot M, Ruidavets J, Marqui J, Esquirol Y, Baracat B, Ferrires J: Environmental factors associated with body mass index in a population of Southern France. Eur J Cardiovasc Prev Rehabil 2004;11:291-297.

10 Najafian J, Toghianifar N, Mohammadifard N, Nouri F: Association between sleep duration and metabolic syndrome in a population-based study: Isfahan Healthy Heart Program. J Res Med Sci 2011;16:801-806.

11 Hsieh S, Muto T, Murase T, Tsuji H, Arase Y: Association of short sleep duration with obesity, diabetes, fatty liver and behavioral factors in Japanese men. Intern Med 2011;50:2499-2502.

12 Börnhorst C, Hense S, Ahrens W, Gebestreit A, Reisch L, Barba G, von Kries R, Bayer O: From sleep duration to childhood obesity - what are the pathways? Eur J Pediatr 2012;171:1029-1038. 
Sayón-Orea et al:: Association between Sleeping Hours and Siesta and the Risk of Obesity: The SUN Mediterranean Cohort

13 Patel SR: Reduced sleep as an obesity risk factor. Obes Rev 2009;10:61-68.

14 Kobayashi D, Takahashi O, Shimbo T, Okubo T, Arioka H, Fukui T: High sleep duration variability is an independent risk factor for weight gain. Sleep Breath 2012;17:167-172.

15 Patel SR, Blackwell T, Redline S,Ancoli-Israel S, Cauley JA, Hillier TA, Lewis CE, Orwoll ES, Stefanick ML, Taylor BC, Yaffe K, Stone KL: The association between sleep duration and obesity in older adults. Int J Obes 2008;32: 1825-1834.

16 Tatone-Tokuda F, Dubois L, Ramsay T, Girard M, Touchette E, Petit D, Montplaisir JY: Sex differences in the association between sleep duration, diet and body mass index: a birth cohort study. J Sleep Res 2012;21:448460.

17 Lopez-Garcia E, Faubel R, Leon-Muñoz L, Zuluaga M, Banegas J, Rodriguez-Artalejo F: Sleep duration, general and abdominal obesity, and weight change among the older adult population of Spain. Am J Clin Nutr 2008; 87:310-316.

18 Patel S, Malhotra A, White D, Gottlieb D, Hu F: Association between reduced sleep and weight gain in women. Am J Epidemiol 2006;164:947-954.

19 Patel S, Hu FB: Short sleep duration and weight gain: a systematic review. Obesity (Silver Spring) 2008;16: 643-653.

20 Hasler G, Buysse DJ, Klaghofer R, Gamma A, Ajdacic V, Eich D, Rössler W, Angst J. The association between short sleep duration and obesity in young adults: a 13-year prospective study. Sleep 2004;27:661-666.

-21 Martínez-González MA, Sanchez-Villegas A, De Irala J, Marti A, Martínez JA: Mediterranean diet and stroke: objectives and design of the SUN project. Seguimiento Universidad de Navarra. Nutr Neurosci 2002;5:65-73.

22 Willett WC.: Issues in analysis and Presentation of dietary data; in Willett WC (ed): Nutritional Epidemiology, 2nd ed. New York, Oxford University Press, 1998, pp 321-345.

-23 Martín-Moreno JM, Boyle P, Gorgojo L, Maisonneuve P, Fernandez-Rodriguez JC, Salvini S, Willet WC: Development and validation of a food frequency questionnaire in Spain. Int J Epidemiol 1993;22:512-519.

24 de la Fuente-Arrillaga C, Ruiz Z, Bes-Rastrollo M, Sampson L, Martinez-Gonzalez M: Reproducibility of an FFQ validated in Spain. Public Health Nutr 2010;13:1364-1372.

25 Mataix J: Tabla de composición de alimentos españoles. 4th ed. Granada, Universidad de Granada, 2003.

26 Moreiras O, Carbajal A, Cabrera L, Cuadrado C: Las tablas; in Moreiras O, Carbajal A, Cabrera L, Cuadrado C (eds): Tabla de composicion de alimentos. 11a. Edición ed. Madrid, Pirámide, 2007, pp 37-46.

27 Bes-Rastrollo M, Pérez-Valdivieso JR, Sanchez-Villegas A, Alonso A, Martinez-Gonzalez MA: Validación del peso e índice de masa corporal auto-declarados de los participantes de una cohorte de graduados universitarios. Rev Esp Obes 2005;3:183-189.

-28 Martinez-Gonzalez MA, López-Fontana C, Varo JJ, Sánchez-Villegas A, Martinez JA: Validation of the Spanish version of the physical activity questionnaire used in the Nurses' Health Study and the Health Professionals' Follow-Up Study. Public Health Nutr 2005;8:920-927.

-29 Hernán M, Hernández Díaz S, Werler M, Mitchell A: Causal knowledge as a prerequisite for confounding evaluation: an application to birth defects epidemiology. Am J Epidemiol 2002;155:176-184.

30 Bes Rastrollo M, Sánchez Villegas A, Gómez Gracia E, Martínez JA, Pajares R, Martínez González M: Predictors of weight gain in a Mediterranean cohort: the Seguimiento Universidad de Navarra Study. Am J Clin Nutr 2006; 83:362-370.

31 Van Cauter E, Knutson K, Leproult R, Spiegel K: The impact of sleep deprivation on hormones and metabolism. Medscape Neurol Neurosurg 2005;7.

-32 Knutson K, Van Cauter E: Associations between sleep loss and increased risk of obesity and diabetes. Ann N Y Acad Sci 2008;1129:287-304.

-33 Sayon-Orea C, Bes-Rastrollo M, Nunez-Cordoba J, Basterra-Gortari FJ, Beunza JJ, Martinez Gonzalez MA: Type of alcoholic beverage and incidence of overweight/obesity in a Mediterranean cohort: the SUN project. Nutrition 2011;27:802-808.

34 Bell JF, Zimmerman FJ: Shortened nighttime sleep duration in early life and subsequent childhood obesity. Arch Pediatr Adolesc Med 2010;164:840-845.

35 Naska A, Oikonomou E, Trichopoulou A, Psaltopoulou T, Trichopoulos D: Siesta in healthy adults and coronary mortality in the general population. Arch Intern Med 2007;167:296-301.

-36 Bursztyn M, Ginsberg G, Hammerman Rozenberg R, Stessman J: The siesta in the elderly: risk factor for mortality? Arch Intern Med 1999;159:1582-1586.

37 Dhand R, Sohal H: Good sleep, bad sleep! The role of daytime naps in healthy adults. Curr Opin Pulm Med 2006; 12:379-382. 